that to every sequence $\alpha_{1}, \alpha_{2}, \ldots$ (with $\alpha_{1}=1$ ) of nonnegative integers there exists exactly one real number in the interval $[0,1)$ to which the given sequence $\alpha_{1}, \alpha_{2}, \ldots$ corresponds.

Mathematisches Institut der Universität Köln, KöLn, Germany.

\title{
A SHORT PROOF OF AN INEQUALITY FOR THE PERMANENT FUNCTION
}

PETER M. GIBSON

Let $A$ be a substochastic matrix, i.e., a square matrix of nonnegative numbers with each row sum no greater than 1 . We have obtained a lower bound for the permanent of $I-A$.

Theorem. If $A$ is a substochastic matrix, then

$$
\text { per }(I-A) \geqq 0 \text {. }
$$

It was brought to our attention by Marcus and Minc [2] that Brualdi and Newman have proved this theorem. Indeed, two proofs of this theorem are contained in a paper that will appear in the Oxford Quarterly [1]. The proof that we shall give, shorter than and quite different from the Brualdi-Newman proofs, shows that this theorem is almost a corollary of the Ryser representation of the permanent.

Let $B$ be an $n$-square matrix and let $B_{r}$ denote a matrix obtained from $B$ by replacing some $r$ columns of $B$ by zero columns. Let $S\left(B_{r}\right)$ be the product of the row sums of the matrix $B_{r}$. Ryser [3] has proved that the permanent of $B$ is given by

$$
\begin{aligned}
\operatorname{per}(B)= & S\left(B_{0}\right)+\sum(-1) S\left(B_{1}\right)+\sum(-1)^{2} S\left(B_{2}\right)+\cdots \\
& +\sum(-1)^{n-1} S\left(B_{n-1}\right),
\end{aligned}
$$

where $\sum(-1)^{r} S\left(B_{r}\right)$ denotes the sum over all $\left(\begin{array}{l}n \\ r\end{array}\right)$ replacements of $r$ of the columns of $B$ by zero columns.

Let $B=I-A$ where $A$ is a substochastic matrix. The $i$ th row sum of $B_{r}$ is nonpositive or nonnegative according to whether the $i$ th column of $B_{r}$ is a zero or a nonzero column. Hence there are at least $r$ row sums of $B_{r}$ that are nonpositive and at least $n-r$ that are nonnegative. Therefore

Received by the editors September 2, 1965. 


$$
\begin{aligned}
(-1)^{r} S\left(B_{r}\right) & \geqq 0, \\
\operatorname{per}(I-A) & =\operatorname{per}(B) \geqq 0 .
\end{aligned}
$$

We are indebted to Morris Newman for a preprint of [1].

Morris Newman informs me that essentially the same proof was communicated to him independently by Hazel Perfect.

\section{REFERENCES}

1. R. A. Brualdi and M. Newman, Proof of a permanental inequality, Quart. J. Math. Oxford Ser. (2) (to appear).

2. M. Marcus and H. Minc, Permanents, Amer. Math. Monthly 72 (1965), 577591.

3. H. J. Ryser, Combinatorial mathematics, Carus Mathematical Monograph No. 14, Mathematical Association of America, 1963.

U. S. NAVAL RESEARCh LABORATORY

North Carolina State University at Raleigh 